\title{
FACTORES ASOCIADOS A LA INEFICACIA DE LA LEY 30364, LEY PARA PREVENIR, SANCIONAR Y ERRADICAR LA VIOLENCIA CONTRA LAS MUJERES Y LOS INTEGRANTES DEL GRUPO FAMILIAR, EN UNA PROVINCIA PERUANA.
}

FACTORS ASSOCIATED WITH THE INEFFICIENCY OF LAW 30364, LAW TO PREVENT, PUNISH AND ERADICATE VIOLENCE AGAINST WOMEN AND MEMBERS OF THE FAMILY GROUP, IN A PERUVIAN PROVINCE.

\author{
Alex Efraín Paco Ale 1 \\ Mario César Gálvez Marquina²
}

Aceptado: 17/09/2019

Publicado online:15/01/2020

\section{RESUMEN}

La investigación buscó establecer si la falta de un equipo multidisciplinario y la falta de colaboración de la víctima estarían asociadas a la ineficacia de la Ley 30364 en la provincia de Jaén. Se analizaron 618 expedientes y se utilizó la técnica de análisis documental. La investigación fue no experimental de diseño transversal de tipo correlacional, de nivel relacional y de tipo básico. Se pudo establecer que al $18,1 \%$ de las víctimas se les realizaron oportunamente el peritaje médico legal y que al $17 \%$ se les practicó el peritaje psicológico de manera oportuna. Así mismo, se pudo establecer que los informe elevados con los documentos necesarios para brindar las medidas de protección provisionales solo son el $26.4 \%$ de los informes estaban completos. Así mismo, se estableció que el $61.7 \%$ de los informes se remitieron de la PNP al Juzgado de familia antes de las 24 horas y que el $4.5 \%$ de las víctimas recibieron medidas de protección antes de las 72 horas. Finalmente, se pudo establecer que la falta de un equipo multidisciplinario y la falta de colaboración de la víctima estaría asociado a la no remisión de los

\footnotetext{
1 Doctor en Derecho. Adjunto superior titular de la Fiscalía Superior Mixta de Jaén, del Distrito Fiscal de Lambayeque-Ministerio Público, Cajamarca, Perú

2 Doctor en Ciencias de la Educación. Director de Antarki sociedad comercial de responsabilidad limitada - Antarki S.R.L, Tacna, Perú
} 
informes de la PNP al Juzgado antes de las 24 horas $(p=0,000)$; a no completar los informes remitidos de la PNP al Juzgado $(p=0,000)$; y a la no emisión de medidas de protección antes de las 72 horas $(p=0,000)$.

Palabras clave: Ley 30364, violencia familiar, eficacia de la Ley.

\begin{abstract}
The research sought to establish the lack of a multidisciplinary team and the lack of collaboration of the person that corresponds to us. The research was not experimental in the transversal design of correlational-causal type, relational level and basic type. It was established that $18.1 \%$ of the victims were treated in a timely manner by the legal medical examination and that $17 \%$ were subjected to a psychological appraisal in a timely manner. Likewise, $26.4 \%$ of the reports are complete. Likewise, it is said that $61.7 \%$ of the reports were sent to the JPP Family Court before 24 hours and that $4.5 \%$ of the responses to the protective measures before 72 hours. Finally, it was established that the lack of a multidisciplinary team and the lack of collaboration of the victim would be associated with the nonreferral of the PNP reports to the Court before 24 hours $(p=, 000)$; not to complete the reports sent from the PNP to the Court $(p=, 000)$; and to the non-issuance of protective measures before 72 hours $(p=$, 000).
\end{abstract}

Keywords: Law 30364, family violence, effectiveness of the Law.

\title{
INTRODUCCIÓN
}

La investigación buscó probar si la falta de un equipo multidisciplinario y la falta de colaboración de la víctima estarían asociadas a la ineficacia de la Ley 30364, Ley para prevenir, sancionar y erradicar la violencia contra las mujeres y los integrantes del grupo familiar, en la provincia de Jaén (Lambayeque-Perú). La violencia familiar es un problema que afecta a muchas familias peruanas, sin embargo, son muy pocas las personas que acuden a denunciarlo. Según datos expuestos en el 2012 por la encuesta nacional demográfica y de salud familiar (ENDES), 4 de cada 10 mujeres fueron víctimas de violencia, encontrándose los índices más altos en la ciudad de Lima en donde 6 de cada 10 mujeres, en el distrito, son maltratadas. El 91\% de las mujeres, según el MINDES, han sido agredidas por sus esposos o convivientes; siendo las formas más comunes de violencia las cachetadas, patadas y jalones de cabello, entre otros. Como consecuencia de las agresiones físicas ocasionadas por algún miembro de su familia, 5 de cada 10 mujeres refirió moretones o heridas. Así mismo, de todas las mujeres encuestadas, el $6.5 \%$ refirió haber tenido una pérdida del embarazo como consecuencia de la agresión. Como se ve, el problema dista mucho de ser resuelto, el Estado con la intensión de poder mitigar el daño de la violencia familiar publicó el 23 de noviembre del 2015, la Ley $N^{\circ} 30364$, "Ley para prevenir, sancionar y erradicar la violencia contra las mujeres y 
los integrantes del grupo familiar", estableciendo en ellas disposiciones que preconizan la celeridad en la recaudación de medios probatorios para garantizar la seguridad de la víctima.

En el Distrito Judicial de Lambayeque, específicamente en la provincia de Jaén, son pocas las denuncias que prosperan a favor de la víctima de violencia familiar, quedando sus casos archivados y esto como se ha podido advertir en muchos casos es por la falta de pruebas. El principal motivo es la falta de colaboración de las víctimas, y otro de los factores, que posiblemente sea una de las causas de este archivamiento, son los cortos plazos que tiene la policía desde la publicación de la Ley $N^{\circ} 30364$. Si bien es cierto, son muchos los factores que se deben analizar para detener la violencia familiar, no es menos cierto que la correcta administración de la justicia para sancionar a los que cometen violencia familiar es imprescindible, pues, es uno de los factores que hacen que los actos de violencia no vuelvan a repetirse. El Estado publicó el 23 de noviembre del 2015 la Ley 30364 que realizó importantes modificaciones en lo que se refiere al procedimiento policial para el recaudo de medios probatorios. Quispe Chata, durante el año 2013, evaluó los factores que facilitan el archivamiento de los casos de denuncias de violencia familiar en el Distrito Judicial de Moquegua. Refiere que el $90 \%$ del archivamiento se dio a la falta de pruebas, que el $68 \%$ de las investigaciones preliminares que se archivaron las realizó la Policía Nacional del Perú y que el principal motivo del archivamiento fue la falta de cooperación de las víctimas y la negligencia de la PNP al momento de recabar los medios probatorios en la investigación preliminar de las denuncias por violencia familiar. Paco Ale, el año 2015, en Jaén, Distrito Judicial de Lambayeque refiere que el principal factor que evita el recaudo de los medios probatorios es la falta de colaboración de las víctimas (70\%), así mismo la celeridad en la recepción y las diligencias que realizan los efectivos de la PNP son lentas. La Ley 30364, Ley para prevenir, sancionar y erradicar la violencia contra las mujeres y los integrantes del grupo familiar, fue publicada el 23 de noviembre de 2015 por el Congreso de la República.

Entre los aspectos más importantes que contempla esta ley se tiene: Tipo de violencia y Violencia contra la mujer o integrantes de grupo familiar. Alcázar y Ocampo (2016), refiere que la violencia tiene una base social. Actualmente, en nuestra sociedad se puede ver que la violencia contra la mujer se manifiesta de distintas maneras y en cualquier esfera social. Cussiánovich, Tello, y Sotelo (2007), afirman que la violencia que sufren las mujeres no es exclusiva de un tipo de organización política o económica.

La Asamblea General de la Organización de los Estados Americanos (1996, pág. 76), promulgó la Ley 24.632 y en su Artículo $1^{\circ}$ expresa que "cualquier acción o conducta que, basada en su condición de género, cause muerte, daño o sufrimiento físico, sexual o psicológico a la mujer, tanto en el ámbito público como en el privado". Para combatir la violencia familiar se promulgó, en el año de 1997, la Ley de Protección frente a la Violencia Familiar (Ley N²6260, 1997), Ley que ya ha sido derogada en el 2015 y entró en vigencia la Ley para prevenir, sancionar y erradicar la violencia contra las mujeres y los integrantes del grupo familiar (Ley $N^{\circ} 30364,2015$ ). 
Gonzales (2017) considera que el establecer el tipo de violencia económica a través de la ley, busca la prevención de cualquier acto que busque menoscabar, a través de los recursos económicos, a cualquier integrante de la familia. Se ha podido establecer que la violencia económica necesita una intención del agresor como elemento subjetivo, que permiten establecer la línea entre una relación violenta y otra que no lo es. Es decir, cuando el agresor restringe la economía de la víctima la intención es menoscabarla (Gonzales, 2017). Para lograr el desarrollo de las organizaciones, se requiere de individuos especializados en otras ramas, es necesario ampliar esta coordinación con el objeto de beneficiar a la sociedad, gracias a la unión de profesionales que colaboran entre sí (Ardines, 2016). Existen tres niveles de interdisciplinariedad de acuerdo a la interacción que se logra entre sus integrantes (Pizarro, 2012), estos son: el trabajo multidisciplinario, interdisciplinariedad y transdisciplinariedad. Da Silva (1964) considera que una ley es eficaz en cuanto tiene una mejor proyección en el medio social, en el que se debe actuar siguiendo las definiciones abstractas que se enriquecen en él. En el marco de las normas jurídicas se pueden clasificar como imperativas o permisivas.

\section{OBJETIVO}

Determinar cuáles son los factores asociados a la ineficacia de la Ley 30364, Ley para prevenir, sancionar y erradicar la violencia contra las mujeres y los integrantes del grupo familiar, en la provincia de Jaén, año 2017.

\section{METODOLOGÍA}

Tipo básica, explicativa, no experimental de diseño transversal. Se desarrolló en el Juzgado de familia de Jaén del distrito judicial de Lambayeque durante el año 2017. Para la presente investigación la unidad de estudio son las denuncias por violencia contra las mujeres y los integrantes del grupo familiar presentado en las comisarías de la provincia de Jaén y elevadas al Juzgado de Familia de Jaén del distrito judicial de Lambayeque, las cuales son 618 casos. Se utilizó la técnica de análisis documental, tanto en las denuncias por violencia contra las mujeres y los integrantes del grupo familiar interpuestos en las comisarías PNP de la provincia de Jaén, como en los expedientes judiciales que contienen las medidas de protección sobre las mismas denuncias.

\section{RESULTADOS}

Para analizar si se cumplieron los plazos establecidos de acuerdo al artículo 15 de la Ley №30364 se contabilizó los días que demoró la PNP para elevar el informe policial al Juzgado de Familia. El $61.7 \%$ de los casos denunciado fueron elevados al Juzgado de Familia en las 24 horas que establece la Ley 30364. Se observa también que el $10.5 \%$ dentro de las 48 horas de conocido el hecho, el $14.9 \%$ dentro de las 72 horas y el $12.5 \%$ después de las 72 horas de haberse conocido el hecho (Tabla 01).

Tabla 1

Demora de la PNP en informar al Juzgado de familia. 


\begin{tabular}{lcc}
\hline $\begin{array}{l}\text { Tiempo Demora de la PNP en informar al Juzgado } \\
\text { de familia }\end{array}$ & $\mathbf{n}$ & $\%$ \\
\hline Antes de las 24 horas. & 381 & 61.70 \\
Entre las 24 y 48 horas. & 65 & 10.50 \\
Entre las 48 y 72 horas. & 92 & 14.90 \\
Más de 72 horas. & 80 & 12.90 \\
Total & 618 & 100.00 \\
\hline
\end{tabular}

Respecto a los informes elevados con los documentos necesarios para brindar las medidas de protección provisionales, solo el $26.4 \%$ de los informes estaban completos. Los informes policiales elevados al Juzgado de Familia de Jaén contienen solo, en el $11.8 \%$, las fichas de valoración de riesgo en mujeres víctimas de violencia de pareja, las cuales son obligatorias de acuerdo a la RD. N 925-A-2016-DIRGEN/EMG-PNP. Guía de procedimiento para intervención de la Policía Nacional en el marco de la Ley $N^{\circ} 30364$ (2016).

En la Tabla 2, se puede apreciar que el $21.8 \%$ de las denuncias presentadas a las comisarías y que fueron elevadas al Juzgado de familia de Jaén fueron archivadas. Según el Artículo 16 de la Ley Nro. 30364, los juzgados de familia, luego de recibir la denuncia policial por violencia familiar tienen un plazo de setenta y dos horas para emitir las medidas de protección provisionales a la víctima. Sin embargo, solo el $4.5 \%$ de las denuncias remitidas al Juzgado de Familia de Jaén cumplen con emitirlas en el plazo establecido por ley. Asimismo, solo el $4.5 \%$ de las resoluciones cumplieron con emitir las medidas de protección antes de las 72 horas y el $73.6 \%$ no lo hicieron. El $100 \%$ de las denuncias contemplaron la prohibición del derecho de tenencia y porte de armas o el inventario sobre sus bienes. El $21.8 \%$ de las denuncias presentadas a las comisarías y que fueron elevadas al Juzgado de familia de Jaén fueron archivadas. Sólo el $4.5 \%$ de las denuncias remitidas cumplen con emitirlas en el plazo establecido por ley. El $4.5 \%$ de las resoluciones cumplieron con emitir las medidas de protección antes de las 72 horas, el $73.6 \%$ no lo hicieron y al $21.8 \%$ de los casos aun no eran emitidas durante la investigación.

El análisis de los expedientes por violencia familiar ha permitido establecer que, del total de denuncias que no fueron archivadas, en el $100 \%$ de estas se pudo hallar como medida provisional el retiro del agresor del domicilio, el impedimento de acercamiento o proximidad a la víctima y la prohibición de comunicación con la víctima.

Sólo el $20.5 \%$ de las medidas de protección provisional emitidas contemplaron la suspensión temporal de visitas del agresor, el $47 \%$ brindaron resguardo y rondas policiales a la víctima, el $32.9 \%$ contempló la prohibición al agresor de acceder a lugares de trabajo o estudio, manteniendo una distancia de 300 metros de la víctima, un $20.3 \%$ prohibieron al agresor el disponer de los bienes comunes conseguidos 
durante la convivencia o el matrimonio y finalmente, el $15.7 \%$ prohibieron al agresor trasladar los bienes comunes conseguidos durante la convivencia o el matrimonio.

Sólo al 18,1\% de los casos sí se les realizó de manera oportuna el peritaje, mientras que al $81.9 \%$ no se lo hizo. Al $17 \%$ se les practicó el peritaje psicológico de manera oportuna, mientras que al $82 \%$ no.

En la Tabla 3 se observa las diligencias policiales que se realizaron de manera oportuna y que se elevaron al Juzgado de Familia dentro de las 24 horas que considera la Ley 30364. 
Tabla 2

Cumplimiento de emisión de medidas de protección

\begin{tabular}{|c|c|c|c|}
\hline \multicolumn{2}{|c|}{ Cumplimiento de emisión de medidas de protección } & $\mathbf{n}$ & $\%$ \\
\hline \multirow{3}{*}{$\begin{array}{l}\text { Estado de los procesos } \\
\text { (Archivados) }\end{array}$} & Sí & 135 & 21.80 \\
\hline & No & 483 & 78.20 \\
\hline & Total & 618 & 100.00 \\
\hline \multirow[t]{11}{*}{ Tiempo } & Menos de 3 días & 28 & 4.50 \\
\hline & Entre 4 y 5 días & 12 & 1.90 \\
\hline & Entre 6 y 7 días & 7 & 1.10 \\
\hline & Entre 8 y 10 días & 28 & 4.50 \\
\hline & Entre 11 y 15 días & 89 & 14.40 \\
\hline & Entre 16 y 30 días & 57 & 9.20 \\
\hline & Entre 31 y 60 días & 176 & 28.50 \\
\hline & Entre 61 y 90 días & 56 & 9.10 \\
\hline & Entre 91 y 120 días & 6 & 1.00 \\
\hline & Más de 120 días & 24 & 3.90 \\
\hline & Total & 483 & 100.00 \\
\hline \multirow{3}{*}{$\begin{array}{l}\text { Resoluciones emitidas } \\
\text { antes de las } 72 \text { hora }\end{array}$} & Sí & 28 & 5.80 \\
\hline & No & 455 & 94.20 \\
\hline & Total & 483 & 100.00 \\
\hline \multirow{3}{*}{$\begin{array}{l}\text { Medidas de protección } \\
\text { interpuestas en todos los } \\
\text { expedientes }\end{array}$} & Retiro del agresor del domicilio. & 483 & 100.00 \\
\hline & $\begin{array}{l}\text { Impedimento de acercamiento a la } \\
\text { víctima. }\end{array}$ & 483 & 100.00 \\
\hline & $\begin{array}{l}\text { Prohibición de comunicación con } \\
\text { la víctima. }\end{array}$ & 483 & 100.00 \\
\hline \multirow{2}{*}{$\begin{array}{l}\text { Medidas de } \\
\text { protección no } \\
\text { interpuestas en } \\
\text { ningún expediente }\end{array}$} & $\begin{array}{l}\text { Prohibición del derecho de } \\
\text { tenencia y porte de armas }\end{array}$ & 483 & $\begin{array}{c}100 . \\
00\end{array}$ \\
\hline & Inventario sobre bienes & 483 & $\begin{array}{c}100 . \\
00\end{array}$ \\
\hline \multirow{3}{*}{$\begin{array}{l}\text { Suspensión } \\
\text { temporal de } \\
\text { visitas }\end{array}$} & Sí & 99 & $\begin{array}{l}20 . \\
50\end{array}$ \\
\hline & No & 384 & $\begin{array}{l}79 . \\
50\end{array}$ \\
\hline & Total & 483 & $\begin{array}{l}78 . \\
20\end{array}$ \\
\hline
\end{tabular}




\begin{tabular}{|c|c|c|c|}
\hline \multirow[t]{3}{*}{$\begin{array}{l}\text { Resguardo } \\
\text { policial }\end{array}$} & Sí & 227 & $\begin{array}{c}47.0 \\
0\end{array}$ \\
\hline & No & 256 & $\begin{array}{c}53.0 \\
0\end{array}$ \\
\hline & Total & 483 & $\begin{array}{c}100 . \\
00\end{array}$ \\
\hline \multirow{3}{*}{$\begin{array}{l}\text { Prohibición de } \\
\text { acceso a } \\
\text { lugares de } \\
\text { trabajo o estudio } \\
\text { a } 300 \text { metros. }\end{array}$} & Sí & 159 & $\begin{array}{c}32.9 \\
0\end{array}$ \\
\hline & No & 324 & $\begin{array}{c}67.1 \\
0\end{array}$ \\
\hline & Total & 483 & $\begin{array}{c}100 . \\
00\end{array}$ \\
\hline \multirow{3}{*}{$\begin{array}{l}\text { Prohibición de } \\
\text { disponer de } \\
\text { bienes comunes }\end{array}$} & Sí & 98 & $\begin{array}{c}20.3 \\
0\end{array}$ \\
\hline & No & 385 & $\begin{array}{c}79.7 \\
0\end{array}$ \\
\hline & Total & 483 & $\begin{array}{c}100 . \\
00\end{array}$ \\
\hline \multirow{3}{*}{$\begin{array}{l}\text { Prohibición de } \\
\text { trasladar bienes } \\
\text { comunes. }\end{array}$} & Sí & 76 & $\begin{array}{c}15.7 \\
0\end{array}$ \\
\hline & No & 407 & $\begin{array}{c}84.3 \\
0\end{array}$ \\
\hline & Total & 483 & $\begin{array}{c}100 . \\
00\end{array}$ \\
\hline
\end{tabular}


Tabla 03

Realización oportuna de las diligencias policiales.

\begin{tabular}{|c|c|c|c|}
\hline \multicolumn{2}{|c|}{ Diligencias policiales } & \multirow{2}{*}{$\frac{n}{586}$} & \multirow{2}{*}{$\begin{array}{l}\% \\
94.80\end{array}$} \\
\hline Declaración de la víctima & Sí & & \\
\hline & No & 32 & 5.20 \\
\hline & Total & 618 & 100.00 \\
\hline \multirow[t]{3}{*}{ Certificación médico legal. } & Sí & 112 & 18.10 \\
\hline & No & 506 & 81.90 \\
\hline & Total & 618 & 100.00 \\
\hline \multirow[t]{3}{*}{ Pericia psicológica } & Sí & 105 & 17.0 \\
\hline & No & 513 & 83.0 \\
\hline & Total & 618 & 100.0 \\
\hline \multirow[t]{3}{*}{ Dosaje etílico } & Sí & 5 & 0.80 \\
\hline & No & 613 & 99.20 \\
\hline & Total & 618 & 100.00 \\
\hline \multirow{6}{*}{$\begin{array}{l}\text { Pericias que no tenían } \\
\text { ningún expediente. }\end{array}$} & Dosaje toxicológico & 618 & 100.00 \\
\hline & Informe de institución de salud & 618 & 100.00 \\
\hline & $\begin{array}{l}\text { Informe de instituciones } \\
\text { públicas }\end{array}$ & 618 & 100.00 \\
\hline & Denuncias previas del agresor & 618 & 100.00 \\
\hline & Incumplimiento alimentario & 618 & 100.00 \\
\hline & Informe SUCAMEC & 618 & 100.00 \\
\hline \multirow[t]{3}{*}{ Declaración de testigo } & Sí & 21 & 3.40 \\
\hline & No & 597 & 96.60 \\
\hline & Total & 618 & 100.00 \\
\hline \multirow{3}{*}{$\begin{array}{l}\text { Antecedentes policiales del } \\
\text { agresor. }\end{array}$} & Sí & 307 & 49.70 \\
\hline & No & 311 & 50.30 \\
\hline & Total & 618 & 100.00 \\
\hline \multirow[t]{3}{*}{ Requisitorias del agresor } & Sí & 179 & 29.00 \\
\hline & No & 439 & 71.00 \\
\hline & Total & 618 & 100.00 \\
\hline Presentación de audios. & Sí & 34 & 5.50 \\
\hline
\end{tabular}




\begin{tabular}{llcc} 
& Total & 618 & 100.00 \\
\hline Presentación de videos & Sí & 21 & 3.40 \\
& No & 597 & 96.60 \\
& Total & 618 & 100.00 \\
\hline Presentación de fotos & Sí & 31 & 5.00 \\
& No & 587 & 95.00 \\
& Total & 618 & 100.00 \\
\hline Presentación de mensajes & Sí & 10 & 1.60 \\
impresos & No & 608 & 98.40 \\
& Total & 618 & 100.00 \\
\hline Incumplimiento alimentario & No & 618 & 100.00 \\
\hline Declaración del & Sí & 128 & 20.70 \\
denunciante & No & 490 & 79.30 \\
& Total & 618 & 100.00 \\
\hline Inspección técnica policial & Sí & 133 & 21.50 \\
& No & 485 & 78.50 \\
& Total & 618 & 100.00 \\
\hline
\end{tabular}

La tabla 3 nos muestra que en el $94.8 \%$ de los informes policiales que se elevaron al Juzgado de Familia de Jaén se consignó la declaración de la víctima, sólo el 18.1\% de los informes cuentan con el peritaje. Únicamente el 17\% de los informes contó con la pericia psicológica al momento de remitirse el informe. Solo el $0,8 \%$ de los informes tenían pericia del dosaje etílico. Solo el 3.4\% contaban declaraciones o manifestaciones de testigos y el $49.7 \%$ contaban con los antecedentes policiales del agresor. El $29 \%$ de los informes contaba con los informes de las requisitorias. el 5.5\% de los informes contenía con la transcripción de audios y solo el $3.4 \%$ con presentación de videos. Respecto a la presentación de fotos solo el $5 \%$ de los informes lo contenía. la presentación de mensaje impresos como prueba de la violencia familiar, como se observa solo el $1.6 \%$ de los informes lo contenía, mientras que el $98.4 \%$ no. El $20.7 \%$ de los informes contaban con la declaración de la persona denunciante, en el caso no haya sido la víctima. Solo el $21.5 \%$ de los informes contenían la inspección técnica policial.

El $15.9 \%$ de las víctimas recibieron oportunamente apoyo legal y tan solo el $9.4 \%$ recibieron apoyo psicológico. El $87.5 \%$ sí acudió de manera oportuna a la pericia médico legal. Del grupo que no acudió, el 15.4\% expuso como razón, no encontrar a personal para que le realice la pericia, el $45.8 \%$ por el exceso personas en espera. 


\section{DISCUSIÓN}

Respecto a la falta de un equipo multidisciplinario asociada a la ineficacia de la Ley 30364, el levantamiento de datos permitió establecer que al 18,1\% de las víctimas se les realizaron oportunamente el peritaje médico legal, mientras que al $81.9 \%$ no se lo hizo. Sobre la realización oportuna del peritaje psicológico se observa que solo al $17 \%$ se les practicó el peritaje de manera oportuna, mientras que al $82 \%$ no. Así mismo, se pudo establecer que los informe elevados con los documentos necesarios para brindar las medidas de protección provisionales solo son el $26.4 \%$ de los informes estaban completos. Finalmente, se pudo establecer que la falta de un equipo multidisciplinario estaría asociada a la no se remisión de los informes de la PNP al Juzgado antes de las 24 horas $(p=, 000)$; a no completar los informes remitidos de la PNP al Juzgado $(p=, 000) ; y$ a la no emisión de medidas de protección antes de las 72 horas $(p=, 000)$.

Sobre la falta de colaboración de la víctima asociada a la ineficacia de la Ley 30364 , se pudo establecer que las víctimas que acudieron de manera oportuna a la pericia médico legal es del $87.5 \%$, mientras que el $12.5 \%$ no lo hizo, sin embargo a pesar de haber acudido oportunamente a la pericia no se les practicó oportunamente al $61.2 \%$ de las víctimas, esto debido a que el $15.4 \%$ no encontró a personal para que le realice la pericia, el $45.8 \%$ acudió pero el exceso personas en espera evitó que se le realice la pericia en el plazo establecido.

Respecto a la pericia psicológica se pudo establecer que el $88.3 \%$ de las víctimas sí acudieron de manera oportuna a la pericia, mientras que el $11.7 \%$ no lo hizo, sin embargo, a pesar de haber acudido oportunamente a la pericia no se les practicó oportunamente al $61,9 \%$ de las víctimas, esto debido a que el $10.8 \%$ no encontró a personal para que le realice la pericia, el 51.1\% acudió, pero el exceso personas en espera evitó que se le realice la pericia en el plazo establecido. Así también, se pudo establecer que el porcentaje de victimas que acudieron oportunamente a las diligencias policiales fue del $26.4 \%$, mientras que el $73.6 \%$ no lo hizo. Sobre el porcentaje de victimas que acudieron oportunamente a las diligencias judiciales, se pudo observar que el $24.3 \%$ acudió oportunamente al total de las diligencias policiales, mientras que el $75.7 \%$ no lo hizo.

Se pudo establecer que la falta de apoyo de la víctima está asociada a la no se remisión de los informes de la PNP al Juzgado antes de las 24 horas $(p=0,000)$; a no completar los informes remitidos de la PNP al Juzgado $(p=0,000)$; y a la no emisión de medidas de protección antes de las 72 horas $(p=0,000)$.

Se respalda la investigación de Quispe (2013) Chata, quien evaluó los factores que facilitan el archivamiento de los casos de denuncias de violencia familiar en el Distrito Judicial de Moquegua. Concluyó que en el $90 \%$ del archivamiento de denuncias por violencia familiar se dio a la falta de pruebas, que el $68 \%$ de las investigaciones preliminares que se archivaron las realizó la Policía Nacional del Perú y que el principal motivo del archivamiento fue la falta de cooperación delas víctimas y las negligencias 
delas víctimas y las negligencias de la PNP al momento de recabar los medios probatorios en la investigación preliminar de las denuncias por violencia familiar. Así mismo, coincidimos con la investigación de Paco (2015), sobre los factores que dificultan el recaudo de medios probatorios en las denuncias por violencia familiar, quien concluyó que en las denuncias que se reciben en las comisarías de Jaén del Distrito Judicial de Lambayeque el principal factor que evita el recaudo de los medios probatorios es la falta de colaboración de las víctimas (70\%), así mismo la celeridad en la recepción y las diligencias que realizan los efectivos de la PNP son lentas.

La falta de un equipo multidisciplinario y la falta de colaboración de la víctima están asociadas a la ineficacia de la Ley 30364. Se recomienda que el distrito fiscal de Lambayeque implemente un sistema multidisciplinario integrado de asistencia a la Policía Nacional del Perú para que realice, dentro de las 24 horas que establece el artículo 15 de la Ley Nro. 30364, los peritajes necesarios para elevar el informe policial al Juzgado de Familia que permita la resolución de las medidas de protección provisionales. Que el Ministerio de la Mujer y Poblaciones Vulnerables diseñe un plan de capacitación a los miembros de la Policía Nacional del Perú sobre la implementación de la Ley Nro. 30364 y su reglamento el DS Nro. 009-2016- MIMP y que, a través de un Decreto Supremo, modifique el artículo 75 del Decreto Supremo $N^{\circ}$ 009-2016-MIMP, Reglamento de la Ley $N^{\circ} 30364$, Ley para prevenir, sancionar y erradicar la violencia contra las mujeres y los integrantes del grupo familiar, de tal forma que se permita, a través de un convenio, la participación de instituciones universitarias y de salud para el apoyo a los equipos multidisciplinarios, especialmente de médicos y psicólogos, para que atiendan directamente en las comisarías a las víctimas que acudan a denunciar casos de violencia en contra de la mujer o los integrantes del grupo familiar y de esta manera la policía pueda cumplir con la recaudación delos medios probatorios dentro de las 24 horas que establece el artículo 15 de la Ley 30364.

\section{REFERENCIAS BIBLIOGRÁFICAS}

Alcázar, L., \& Ocampo, D. (2015). Consecuencias de la violencia doméstica contra la mujer sobre el progreso escolar de los niños y niñas del Perú: Un estudio a nivel nacional diferenciando por género. Lima: Consorcio de investigación económica y social.

Alcázar, L., \& Ocampo, D. (2016). Consecuencias de la violencia doméstica contra la mujer en el progreso escolar de los niños y niñas del Perú. Lima: Grupo de Análisis para el Desarrollo (GRADE).

Ardines, P. (24 de julio de 2016). El trabajo multidisciplinario, nuevo enfoque para la solución de problemas y para la realización de programas en el área de la salud. Obtenido de http://www.posgrado.unam.mx/publicaciones/ant_omnia/ 11/14.pdf

Asamblea General de la Organización de los Estados Americanos. (1996). Convención Interamericana para Prevenir, Sancionar y Erradicar la Violencia contra la Mujer "Convención de Belém do Pará". Recuperado el 19 de Septiembre de 2017, de Ley 24.632 https://www.unicef.org/argentina/spanish/ar_insumos_ConvencionBelem. pdf 
Calvo, R. (2007). La ineficacia de las normas jurídicas en la teoría pura del Derecho. Revista Isonomía de la Universidad de Girona, 171-191.

Congreso de la República del Perú. (2015). Protocolo de identificación, atención y referencia de casos de violencia dentro del sistema educativo nacional. Recuperado el 28 de septiembre de 2017, de http://www2.congreso.gob.pe/ sicr/cendocbib/con4_uibd.nsf/E13D200D63 83837A05257CD000797692/\$FILE/ 33_pdfsam_Guate_Identificacion_de_Casos_de_Violencia_en_EScuelas.pdf

Cussiánovich, A., Tello, J., \& Sotelo, M. (2007). Violencia intrafamiliar. Lima: Unidad de Coordinación del Proyecto Mejoramiento de los Servicios de Justicia.

Cussiánovich, A., Tello, J., \& Sotelo, M. (2007). Violencia intrafamiliar. Lima: Unidad de Coordinación del Proyecto Mejoramiento de los Servicios de Justicia.

Da Silva, A. (1964). Principio del proceso de formación de las leyes en el derecho constitucional. Sao Paulo.

Decreto Supremo Nro. 009-2016-MIMP. (26 de julio de 2016). Decreto Supremo que aprueba el Reglamento de la Ley $\mathrm{N}^{\circ} 30364$, Ley para prevenir, sancionar y erradicar la violencia contra las mujeres y los integrantes del grupo familiar.

Exp. N. ${ }^{\circ}$ 0012-2010-PI/TC. (11 de noviembre de 2011). Sentencia del Tribunal Constitucional del Perú. Lima, Perú.

Gonzales, M. (2017). Violencia contra la mujer en el Distrito de Santiago de Surco. Lima: Fondo Universidad Ricardo Palma.

González, L. (2016). El problema de violencia y sus enfoques. Revista ALAI.

Hanson, C., \& Spross, J. (2005). Advanced practice nursing: An integrative approach. San Luis: Elsevier Saunders.

INEI. (2015). Violencia familiar. En Crecimiento Económico, Población, Características Sociales y Seguridad Ciudadana en la Provincia Constitucional del Callao. (págs. 127-132). Lima. Recuperado el 19 de Septiembre de 2017, de https:// www.inei.gob.pe/media/MenuRecursivo/publicaciones_digitales/Es t/Lib1339/ cap05.pdf

Instituto de Medicina Legal. (2011). Guía de valoración del daño psíquico en víctimas adultas de violencia familiar, sexual, tortura y otras formas de violencia intencional. Lima: Fiscalía de la nación.

Instituto Nacional de Estadística e Informática. (2014). Perfil Sociodemográfico del Cerro San Cosme. Lima: INEI.

Ley $\mathrm{N}^{\circ}$ 26260. (25 de junio de 1997). Ley de protección frente a la violencia familiar. Lima, Perú.

Ley $\mathrm{N}^{\circ}$ 30364. (23 de noviembre de 2015). Ley para prevenir, sancionar y erradicar la violencia contra las mujeres y los integrantes del grupo familiar. Lima, Perú.

Mejía, U., Bolaños, J., \& Mejía, A. (2015). Lesiones por violencia familiar en el reconocimiento médico Legal (RML). Revista Horizonte Médico, 21-29.

Pizarro, R. (11 de agosto de 2012). Los equipos interdisciplinarios. Obtenido de http:// www.binasss.sa.cr/bibliotecas/bhp/cupula/v8n17/art3.pdf

Policía Nacional del Perú. (12 de septiembre de 2016). Guía de procedimientos para la 
para la intervención de la Policía Nacional en el marco de la Ley $N^{\circ} 30364$. Lima.

Torres, E. (2016). La violencia en los Andes. Historia de un concepto, siglos XVI-

XVII. Lima: Fondo Editorial PUCP. 